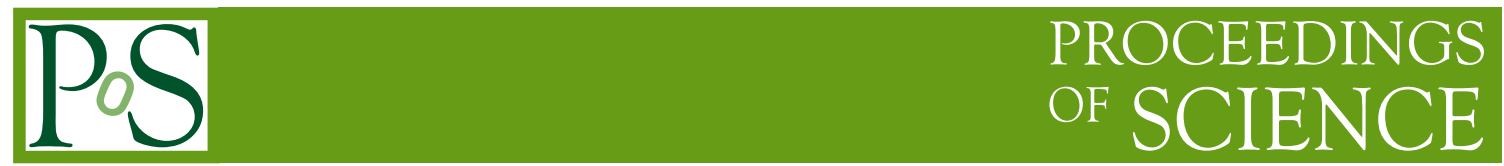

\title{
Higgs bosons of R-symmetric supersymmetric theories
}

\section{Jan Kalinowski*}

Faculty of Physics, University of Warsaw, ul. Hoza 69, 00681 Warsaw, Poland

E-mail: jan.kalinowski@fuw.edu.pl

The Higgs sector of the minimal $R$-symmetric supersymmetric standard model is briefly presented, including the expectations for the production of $R$-Higgs bosons at the LHC and $e^{+} e^{-}$ colliders.

The 2011 Europhysics Conference on High Energy Physics-HEP 2011,

July 21-27, 2011

Grenoble, Rhône-Alpes France

*Speaker. 


\section{Introduction}

At this conference we have seen how new results from Atlas and CMS collaborations have substantially advanced the exclusion limits on supersymmetric particles [1]. In spite of this impressive progress, however, the theoretical arguments for $\mathrm{TeV}$-scale supersymmetry have not yet been invalidated.

Even before the new LHC data, supersymmetry has been under increasing pressure from data on flavor, CP, dark matter, as well as from direct searches at Tevatron. On the theory side, an attractive way of easing the pressure is provided by imposing a continuous $U(1)$ symmetry on the Minimal Supersymmetric Standard Model (MSSM), the $R$-symmetry [2], which removes some of the phenomenologically embarrassing parameters of the theory. Assigning the $R$ charges to the MSSM superfields as in Table 1 (the $R$-charge of the fermionic coordinate $\theta$ is taken as +1 ), the $\mu$ term and baryon- and lepton-number changing terms in the superpotential as well as soft-supersymmetry breaking Majorana gaugino masses and trilinear $A$-terms are forbidden, while Majorana neutrino masses can be generated. However, to give the gaugino and higgsino masses the superfield content of the model has to be extended. The Minimal $R$-symmetric Supersymmetric Standard Model (MRSSM) [3, 4] incorporates (i) new chiral superfields $\hat{\Sigma}_{K}=\left\{\sigma_{K}, \tilde{G}_{K}^{\prime}\right\}$ in the adjoint representation of the gauge group (with $K=C, I, Y$ for $S U(3), S U(2), U(1)$ respectively) (ii) two new iso-dublet chiral superfields $\hat{R}_{u}, \hat{R}_{d}$ (R-Higgs). Then the standard gauginos $\tilde{G}$ are combined with the new ones $\tilde{G}^{\prime}$ to form Dirac fermions $\tilde{G}_{D}=\tilde{G} \oplus \tilde{G}^{\prime}$, while the new Higgses allow to build $R$-symmetric $\mu$-type terms, $\mu_{d} \hat{H}_{d} \cdot \hat{R}_{d}+\mu_{u} \hat{H}_{u} \cdot \hat{R}_{u}$.

The Dirac nature of gauginos as well as new $R_{u, d}$ and adjoint scalar fields $\sigma_{K}$ have far reaching consequences on supersymmetric particle production at the LHC and $e^{+} e^{-}$colliders, cold dark matter expectations and flavor- and CP-changing processes . Here we briefly discuss the experimental footprint of the $R$-symmetric Higgs sector at the LHC and $e^{+} e^{-}$colliders [4].

\section{The Higgs sector}

In addition to the standard Yukawa and new $\mu$-type terms the $R$-symmetry also admits trilinear terms for isospin and hypercharge interactions in the superpotential $\lambda_{d}^{I, Y} \hat{H}_{d} \cdot \hat{\Sigma}_{I, Y} \hat{R}_{d}+\lambda_{u}^{I, Y} \hat{H}_{u}$. $\hat{\Sigma}_{I, Y} \hat{R}_{u}$. The soft-supersymmetry breaking parameters, consistent with $R$-symmetry, include the $B_{\mu}$ for the standard Higgs bosons, soft scalar masses $m_{H_{i}}, m_{R_{i}}$ for $H$ and $R$ Higgses $(i=u, d)$, Dirac gaugino masses $M_{K}^{D}$ and soft masses $M_{\sigma_{K}}$ of the adjoint scalars.

The Higgs potential derived from the superpotential and the soft terms is in general very complex due to the presence of $H, R$ and $\sigma_{I, Y}$ states. Assuming the mass parameters in the $\sigma_{I, Y}$ sector

\begin{tabular}{|l|c||l|c|}
\hline Superfield & $R$-charge & Superfield & $R$-charge \\
\hline matter $\hat{L}, \hat{E}^{c}, \hat{Q}, \hat{D}^{c}, \hat{U}^{c}$ & +1 & gauge chiral $\hat{\Sigma}_{K}$ & 0 \\
gauge vector $\hat{G}_{K}$ & 0 & $R$-Higgs $\hat{R}_{d, u}$ & +2 \\
Higgs $\hat{H}_{d}, \hat{H}_{d}$ & 0 & & \\
\hline
\end{tabular}

Table 1: The R-charges of the MSSM and of the new gauge chiral $\hat{\Sigma}_{K}$ and R-Higgs superfields. 

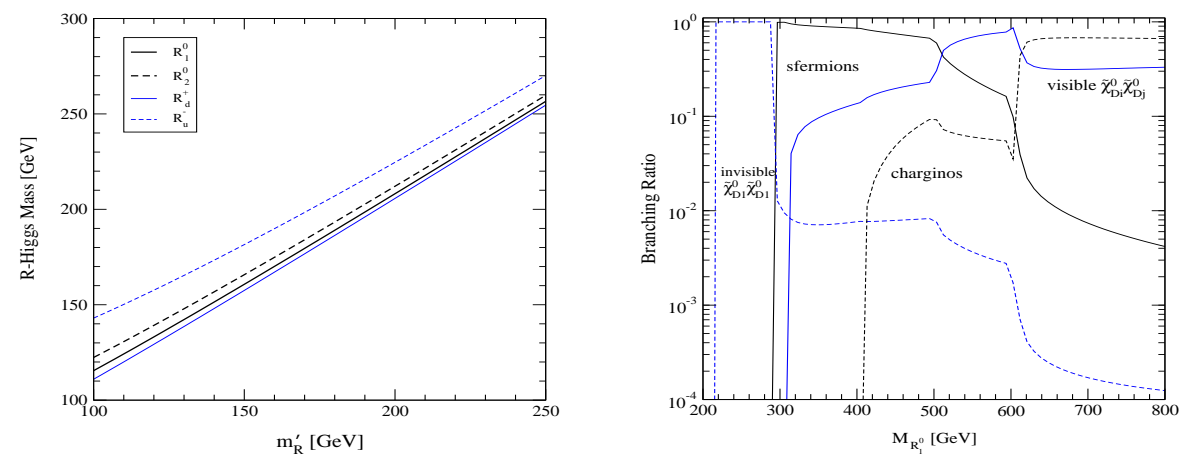

Figure 1: Left: Masses of $R_{1,2}^{0}$ and $R_{d, u}^{ \pm}$Higgs bosons for a common mass parameter $m_{R}^{\prime}=\left(m_{R_{d, u}}^{2}+\mu_{d, u}^{2}\right)^{1 / 2}$. Right: Branching ratios for decays of the neutral $R_{1}^{0}$-Higgs to sfermions, neutralinos and charginos.

large, ${ }^{1}$ of TeV order (to suppress the vacuum expectation value of the iso-vector $\sigma_{I}$, as demanded by the $\rho$ parameter), the neutral part of the potential simplifies to

$$
\begin{aligned}
V= & \left(m_{H_{d}}^{2}+\mu_{d}^{2}\right)\left|H_{d}^{0}\right|^{2}+\left(m_{H_{u}}^{2}+\mu_{u}^{2}\right)\left|H_{u}^{0}\right|^{2}+\left(m_{R_{d}}^{2}+\mu_{d}^{2}\right)\left|R_{d}^{0}\right|^{2}+\left(m_{R_{u}}^{2}+\mu_{u}^{2}\right)\left|R_{u}^{0}\right|^{2}-\left(B_{\mu} H_{d}^{0} H_{u}^{0}+\text { h.c. }\right) \\
& +\left|\lambda_{d}^{I} H_{d}^{0} R_{d}^{0}+\lambda_{u}^{I} H_{u}^{0} R_{u}^{0}\right|^{2}+\left|\lambda_{d}^{Y} H_{d}^{0} R_{d}^{0}-\lambda_{u}^{Y} H_{u}^{0} R_{u}^{0}\right|^{2}+\frac{1}{8}\left(g^{2}+g^{2}\right)\left(\left|H_{d}^{0}\right|^{2}-\left|H_{u}^{0}\right|^{2}-\left|R_{d}^{0}\right|^{2}+\left|R_{u}^{0}\right|^{2}\right)^{2}
\end{aligned}
$$

Since no $B_{\mu}$-type term for the $R$-Higgses is allowed, as required by $R$-symmetry, the $R$-Higgs fields $R_{d, u}$ do not develop non-zero vacuum expectation values. As a result, the $R$ and $H$ Higgs fields do not mix with the other. As the same-sign $R_{u, d}^{+}$carry opposite $R$-charge, they do not mix either, while the mass-eigenstates of the neutral ones are obtained by a standard diagonalization of $2 \times 2$ mass matrix. For $\left(m_{R_{d, u}}^{2}+\mu_{d, u}^{2}\right)^{1 / 2} \equiv m_{R}^{\prime} \geq v$, in analogy to the heavy Higgs bosons of the MSSM, the neutral and charged $R$-Higgses are roughly mass-degenerate, see fig.1 [4], modulo terms of order $g^{2} v^{2} / m_{R}^{\prime}\left(N=2\right.$ susy values of the $\lambda^{I, Y}$ couplings are assumed, and $v=\left(v_{1}^{2}+v_{2}^{2}\right)^{1 / 2}$ is the vev of the MSSM $H$-Higgs fields).

The conserved $R$-charge restricts the $R$-Higgs boson trilinear couplings to pairs of sfermions, $R \tilde{\ell} \tilde{\ell}, R \tilde{q} \tilde{q}$, and chargino/neutralino combinations, $R \tilde{\chi} \tilde{\chi}$; the couplings to pairs of SM particles and Higgs bosons, $R f f, R V V, R H H$ vanish. Therefore, the $R$-Higgs bosons, if kinematically allowed, decay to sfermion pairs, e.g. $R_{d}^{+} \rightarrow \tilde{u}_{L} \tilde{d}_{R}^{*}, \tilde{v}_{L} \tilde{l}_{R}^{*}$, and pairs of charginos/Dirac neutralinos, $R_{d}^{+} \rightarrow \tilde{\chi}_{d}^{+} \tilde{\chi}_{D}^{0} ; R_{d}^{0}$ and $R_{u}^{+, 0}$ have similar decay channels. Fermions, charginos and neutralinos will eventually decay through cascades to the lightest stable neutralino $\tilde{\chi}_{D 1}^{0}$. Note that the light neutral $R$-Higgs bosons may have invisible decay mode if the only open decay channel is $\tilde{\chi}_{D 1}^{0} \tilde{\chi}_{D 1}^{0}$. This is shown in the right panel of fig.1 [4], where a set of 2-body on-shell neutral $R_{1}^{0}$ Higgs decays is displayed (for SPS1a' scenario [6] with the gauginos taken as Dirac and sfermion mixing removed).

\section{3. $R$-Higgs bosons at colliders}

The conserved $R$-charge implies that the $R$-Higgs bosons can be produced only in pairs at the $p p$ collider LHC, via Drell-Yan mechanism, and in $e^{+} e^{-}$annihilation at prospective linear colliders. Fig.2 (from [4]) shows the expected size of the cross sections for the production of

\footnotetext{
${ }^{1}$ The colored scalars, sgluons $\sigma_{C}$ can nevertheless be rather light and copiously produced at the LHC [5].
} 

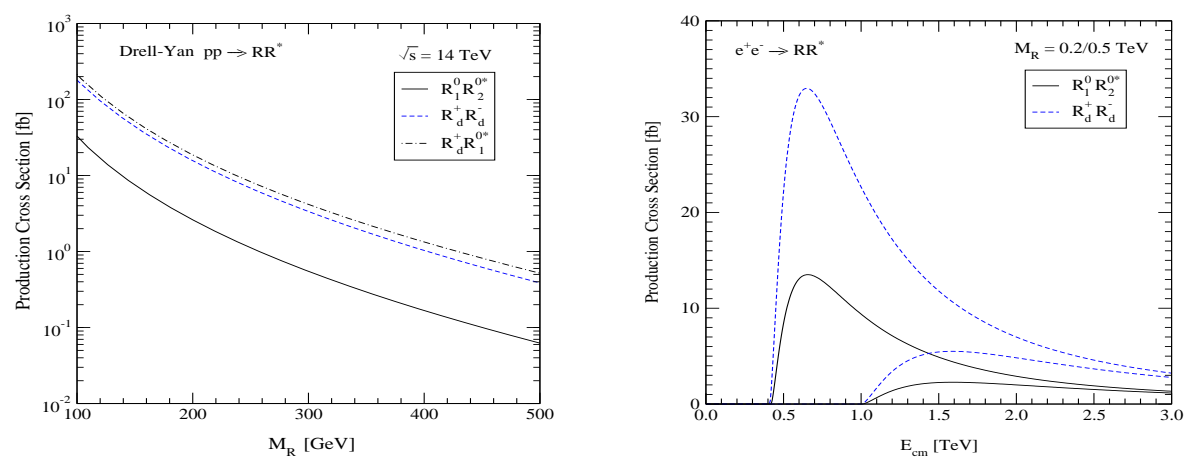

Figure 2: Drell-Yan production of R-Higgs boson pairs at the $14 \mathrm{TeV}$ LHC versus the averaged mass of the produced particles $M_{R}$; Right: Production of R-Higgs boson pairs at $e^{+} e^{-}$colliders for two mass values: $0.2 \mathrm{TeV}$ and $0.5 \mathrm{TeV}$.

neutral/charged $R$-Higgs pairs, $R_{1}^{0} R_{2}^{0^{*}}, R_{d}^{+} R_{d}^{-}$and $R_{d}^{+} R_{1}^{0^{*}}$; cross sections for the diagonal neutral $R$-Higgs boson pairs, $R_{1}^{0} R_{1}^{0^{*}}$ and $R_{2}^{0} R_{2}^{0^{*}}$, vanish for the common $R$-Higgs mass parameter $m_{R}^{\prime}$. Additional sources of $R$ Higgs bosons, though in general at reduced levels, are provided by the fusion channel $p p \rightarrow \gamma \gamma \rightarrow R^{+} R^{-}$, or from heavy MSSM Higgs decays $H \rightarrow R R^{*}$.

Although the details of the experimental signature depend on the specific scenario, the pairproduction of $R$-Higgs bosons determines the characteristic features. Taking again the SPS1a' scenario, we may expect

$$
R^{0} R^{0^{*}} \rightarrow \tau^{+} \tau^{-} \tau^{+} \tau^{-}+\tilde{\chi}_{D 1}^{0} \tilde{\chi}_{D 1}^{0} \tilde{\chi}_{D 1}^{0 c} \tilde{\chi}_{D 1}^{0 c}
$$

final states as a result of the cascade decays: $R^{0} \rightarrow \tilde{\chi}_{D 1}^{0} \tilde{\chi}_{D 2}^{0}$ followed by $\tilde{\chi}_{D 2}^{0} \rightarrow \tau \tilde{\tau}$ followed by $\tilde{\tau} \rightarrow \tau \tilde{\chi}_{D 1}^{0}$. The $\tau^{\prime}$ s give rise to $e, \mu$ leptons and narrow hadronic jets. The four $L S P^{\prime}$ s generate a large amount of missing energy in $e^{+} e^{-}$collisions and missing transverse momentum in proton collisions. Other charge configurations and decay channels of the $R$ pairings have similar characteristics. Thus, the multi-fold $\tau$-multiplicity in association with high values of missing energy/transverse momentum offers promising signatures for detecting $R R$ events.

Acknowledgments: I thank S.Y. Choi, D. Choudhury, A. Freitas, and P. Zerwas for an enjoyable and fruitful collaboration. I also thank DESY for hospitality where this writeup has been completed.

\section{References}

[1] D. Charlton (Atlas), G. Tonelli (CMS), these Proceedings and references therein.

[2] A. Salam and J. A. Strathdee, Nucl. Phys. B 87 (1975) 85. P. Fayet, Nucl. Phys. B 90 (1975) 104.

[3] G. D. Kribs, E. Poppitz and N. Weiner, Phys. Rev. D 78 (2008) 055010.

[4] S. Y. Choi, D. Choudhury, A. Freitas, J. Kalinowski, P. M. Zerwas, Phys. Lett. B697, 215-221 (2011).

[5] S. Y. Choi, M. Drees, J. Kalinowski, J. M. Kim, E. Popenda and P. M. Zerwas, Phys. Lett. B 672 (2009) 246.

[6] J. A. Aguilar-Saavedra et al., Eur. Phys. J. C 46 (2006) 43. 